\title{
Regioisomeric Effect on the Excited-State Fate Leading to Room-Temperature Phosphorescence or Thermally Activated Delayed Fluorescence in a Dibenzophenazine- Cored Donor-Acceptor-Donor System
}

\author{
Takumi Hosono, ${ }^{a}$ Nicolas Oliveira Decarli, ${ }^{b}$ Paola Zimmermann Crocomo, ${ }^{b}$ Tsuyoshi Goya, ${ }^{c}$ Leonardo Evaristo de Sousa, ${ }^{d}$ \\ Norimitsu Tohnai, ${ }^{a}$ Satoshi Minakata, ${ }^{a}$ Piotr de Silva, ${ }^{d *}$ Przemyslaw Data, ${ }^{\text {b* }}$ and Youhei Takeda ${ }^{a *}$ \\ Exploring design principle for switching thermally activated dealyed fluorescecne (TADF) and room temperature phosphorescence (RTP) is a fundamentally \\ imporant research in developing triplet-mediated photofunctional organic materials. Herein systematic studies on the regioisomeric and substituents effects \\ in a twisted donor-acceptor-donor ( $D-A-D)$ scaffold ( $A$ = dibenzo[a,j]phenazine; $D=$ dihydrophenazasiline) on the fate of the excited state have been \\ performed. The study revealed that the regiosiomerism clearly affects the emission behavior of the D-A-D compounds. Distinct difference in TADF, dual TADF \\ \& RTP, and dual RTP were observed, depending on the host used. Furthermore, OLED organic light-emitting diodes (OLEDs) fabricated with the developed \\ emitters achieved high external quantum yields for RTP-based OLEDS up to $7.4 \%$.
}

\section{Introduction}

Heavy-atom-free organic compounds that display thermally activated delayed fluorescence (TADF) and/or roomtemperature phosphorescence (RTP) have emerged as promising emissive materials for efficient organic light-emitting diodes (OLEDs), ${ }^{1-5}$ due to the capability of harvesting electrically-generated excitons. Also, they have increasingly attracted much attention in the fields of anti-counterfeiting, ${ }^{6}$ bio-imaging, theranostics, ${ }^{7}$ and some others. ${ }^{8}$ From a mechanistic point of view, TADF and RTP phenomena are connected through a triplet excited state:9-12 TADF arises through a radiative channel from the singlet excited state $\left(S_{1}\right)$ by recycling $S_{1}$ by way of an energetically-close lying triplet excited state $\left(T_{n}\right.$; the singlet-triplet energy gap $\left.\Delta E_{S T}<c a .0 .3 \mathrm{eV}\right)$, while RTP yields through a radiative channel from $T_{1}$ to the ground state $\left(\mathrm{S}_{0}\right)$. With a moderate $\Delta E_{\mathrm{ST}}(c a .0 .3-0.6 \mathrm{eV})$, reverse intersystem crossing ( $\mathrm{rISC}$ ) to yield the $S_{1}$ and radiative pathway going back to the $S_{0}$ (i.e., RTP) are competing with each other,

\footnotetext{
Department of Applied Chemistry, Graduate School of Engineering, Osaka University, Yamadaoka 2-1, Suita, Osaka 565-0871, Japan.

E-mail: takeda@chem.eng.osaka-u.ac.jp

b. Faculty of Chemistry, Silesian University of Technology, M. Strzody 9, 44-100 Gliwice, Poland.

E-mail: przemyslaw.data@polsl.pl

c. New Business Commercialization Project, NIPPON SHOKUBAI CO. LTD., 5-8 Nishi Otabi-cho, Suita, Osaka 564-0034, Japan

d. Department of Energy Conversion and Storage, Technical University of Denmark, Anker Engelunds Vej 301, 2800 Kongens Lyngby, Denmark.

E-mail: pdes@dtu.dk

Electronic Supplementary Information (ESI) available: experimental procedures for the syntheses of materials, spectroscopic data of new compounds, single crystal Xray crystallographic data, UV-Vis absorption and photoluminescence spectra, thermogravimetric analysis (TGA) profiles, cyclic voltammograms, theoretical calculation details, and the copies of NMR spectra of new compounds.
}

and thereby dual emission of TADF and RTP are observed. ${ }^{13-15}$ Dual emissive organic materials can find in applications such as sensors, data encryption, white-emitting OLEDs (WOLEDs). ${ }^{16}$ Therefore, the regulation of the fate of the triplet excited state leading to TADF or RTP or both by molecular design is fundamentally important in materials chemistry.

Recently, we have successfully developed a heavy-atomfree organic RTP material 1 (Fig. 1) based on a donor-acceptordonor (D-A-D) scaffold $[\mathrm{D}=$ Si,Si-diphenyldihydrophenazasiline (Ph-DHPAS); $\mathrm{A}=$ dibenzo[ $a, j]$ phenazine (DBPHZ)]. ${ }^{17}$ The D-A-D compound is featured with unexpected dual room-temperature phosphorescence (RTP) from $T_{1}\left({ }^{3} L_{1}\right)$ and $\mathrm{T}_{2}\left({ }^{3} \mathrm{CT}_{2}\right)$ in a host matrix, which would be accelerated through a thermally activated reverse internal conversion (TArIC) process. ${ }^{18}$ Utilizing the compound as the emissive material for organic light-emitting diodes (OLEDs), an efficient electrophosphorescence without heavy atoms such as Ir and Pt was achieved. Due to the scarcity of the examples of RTP materials based on the TArIC mechanism, the systematic structure-property relationship study (SPR) is indispensable to cultivate new materials space of heavy-atom-free RTP materials.

Herein we disclose a SPR study of dual RTP materials using the $D-A-D$ derivatives $\mathbf{2}$ and $\mathbf{3}$, which have varied substituents on the silicon center, and their regioisomeric counterparts 4-6 (Fig. 1). Interestingly, the regioisomerism mainly plays an important role in switching the emissive channel of the DBPHZcored D-A-D compounds between TADF and RTP. Although the alternation of substituents on the $\mathrm{Si}$ center less influences the emissive excited-state energy, the thermal stability was significantly affected by the substituent. 


\section{Results and discussion}

3,11-regioisomers

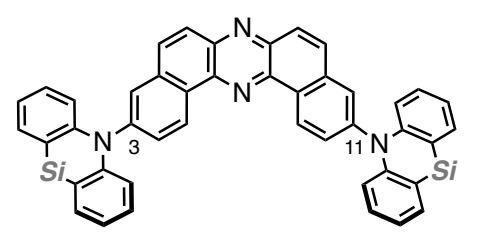

1: $\mathrm{Si}=\mathrm{SiPh}_{2}$ (previous work) 2: $\mathrm{Si}=\mathrm{Si}(i-\mathrm{Pr})_{2}$

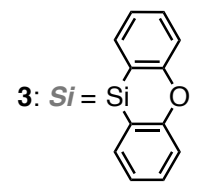

2,12-regioisomers

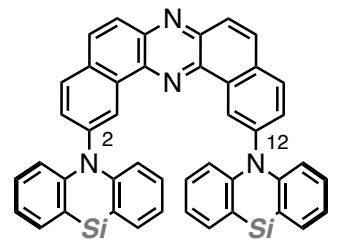

4: $\mathrm{Si}=\mathrm{SiPh}_{2}$ 5: $\mathrm{Si}=\mathrm{Si}(i-\mathrm{Pr})_{2}$

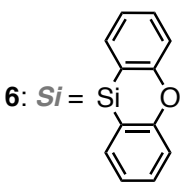

Fig. 1 Chemical structures of materials used in this study.

\section{Material design and synthesis}

To reveal the effect of substituents on the $\mathrm{Si}$ atom and regioisomerism of $\mathbf{1}$ on its physicochemical properties, a series of D-A-D compounds 2-6 were designed (Fig. 1). The synthesis of the $D-A-D$ compounds were conducted in a similar manner with that for 1 (Scheme 1, for the detailed procedures, see the ESI). ${ }^{17}$ The 3,11-dibromo-dibenzophenazine 8, which was prepared through an oxidative skeletal rearrangement of dibromo binaphthalenediamine (BINAM) 7,19 was subjected to the Pd-catalyzed Buchwald-Hartwig double amination with dihydrophenazasilines $\mathbf{9}$ and $\mathbf{1 0}$ to afford the corresponding DA-D compounds $\mathbf{2}$ and $\mathbf{3}$ in good yields, respectively (Scheme $\mathbf{1}$, the upper equations). For the synthesis of 2,12-regioisomers, a new dibromo dibenzophenazine $\mathbf{1 2}$ was synthesized in a good yield, by applying dibromo BINAM 11 to our oxidative skeletal rearrangement reaction conditions (Scheme 1). ${ }^{19}$ The subsequent Pd-catalyzed double amination of 12 with dihydrophenazasilines gave the corresponding 2,12regioisomeric D-A-D compounds 4-6 in good yields (Scheme 1, the bottom equations).

\section{X-Ray crystallographic analysis}

Single crystals of regioisomers $\mathbf{3}$ and $\mathbf{6}$ suitable for X-ray crystallographic analysis were successfully obtained by slow evaporation of bilayer solutions of $n$-hexane $/ \mathrm{CHCl}_{3}$ (Fig. 2, for the detailed crystallographic data, see the Table S1 and S2 in the ESI). The crystallographic analysis revealed that both $D-A-D$ molecules take equatorial-equatorial (eq-eq) type conformation in which two spiro-dihydrophenazasiline donors are connected with the acceptor in a perpendicular fashion (Fig. $2 a, b, e$, and $f$ ). In our previous work on 3,11-isomer 1, theoretical calculations suggested that eq-eq conformer is the most thermodynamically stable one over the other possible conformers (i.e., eq-ax and ax-ax). ${ }^{17}$ Therefore, the eqpreference of dihydrophenazasiline donor in the 3,11disubstituted DBPHZ-cored D-A-D scaffold is reinforced experimentally. Due to the cruciform structure of the spiro motif, the planes of DBPHZ, phenazasiline, and phenoxasilin ring in $\mathbf{3}$ are almost perpendicular to each other (Fig. $2 b$ and c). There is almost no $\pi-\pi$ contact between the adjacent molecule in the packing structure (Fig. $2 d$, interplane distance $=7.75 \AA$ ), probably due to the cruciform molecular architecture. In contrast to the $C_{2 v}$ molecular geometry of $\mathbf{3}$ (Fig. 2e), the 2,12regioisomer 6 takes a less symmetrical conformation, with the DBPHZ core taking a helical structure and the phenazasiline unit taking slightly bent geometry (Fig. 2f). Even though the sterically-congested environment around the D-A connecting positions, the acceptor occupies the equatorial position on the both nitrogen atoms of the donors, and thereby, the two donors take slipped cruciform conformation (Fig. $2 \mathrm{f}$ and g). In contrast to 3,11-regioisomer 3, 2,12-isomer 6 stacks at the DBPHZ unit in

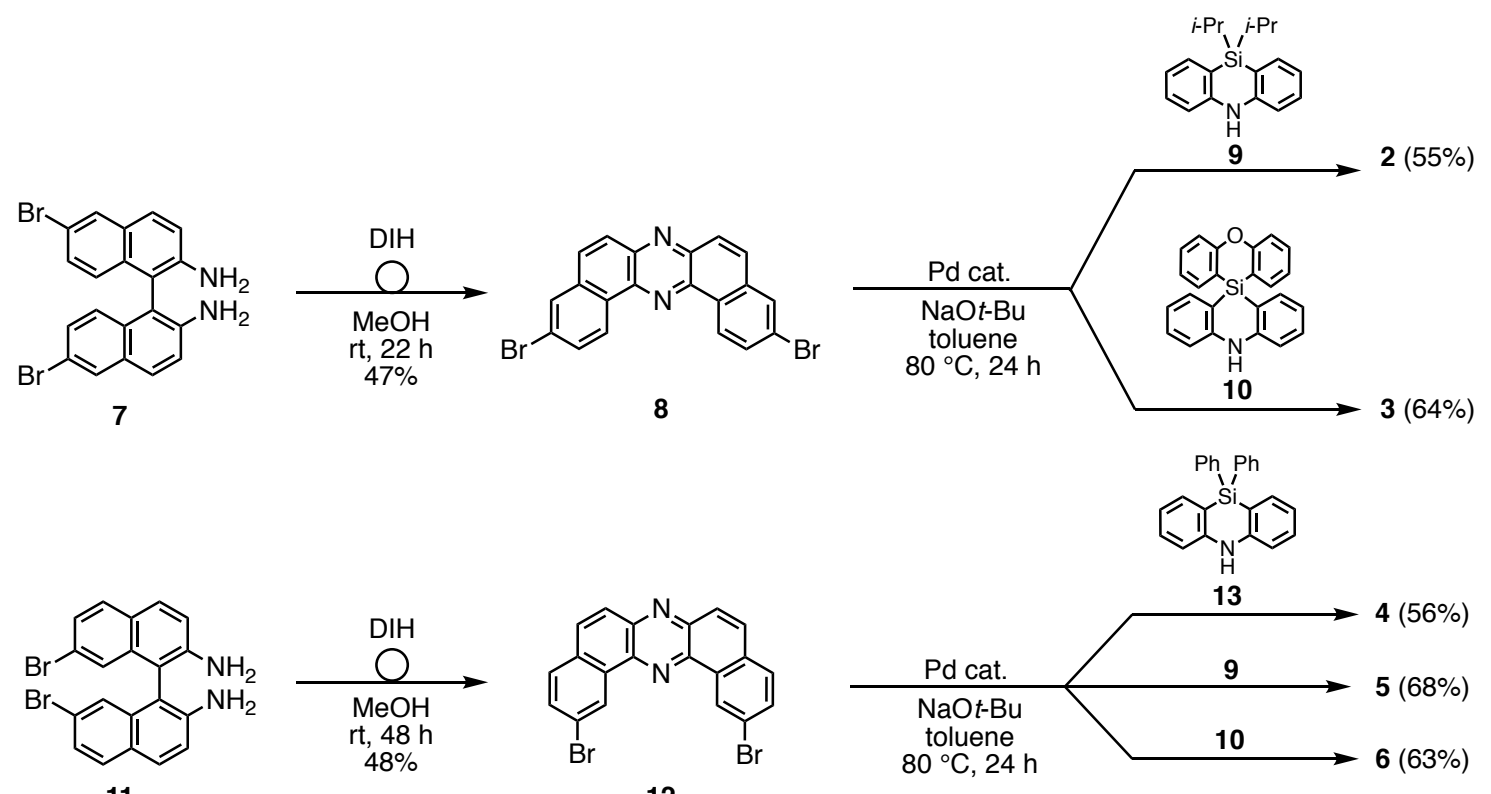

Scheme 1 Synthetic route to 2-6. 
an anti-parallel manner, with the interplane distance of the plane of DBPHZ unit being $3.64 \AA$ (Fig. 2h).
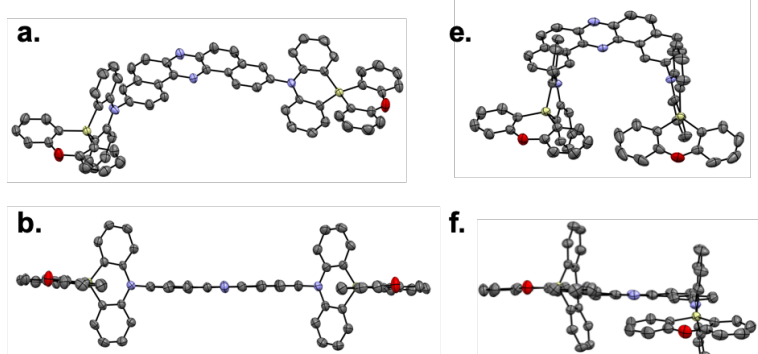

f.

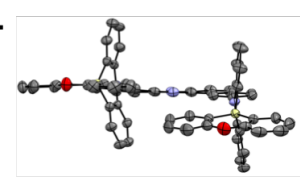

c.

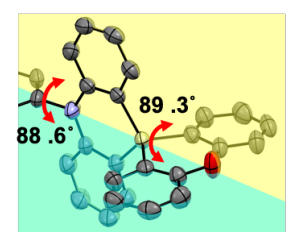

d.

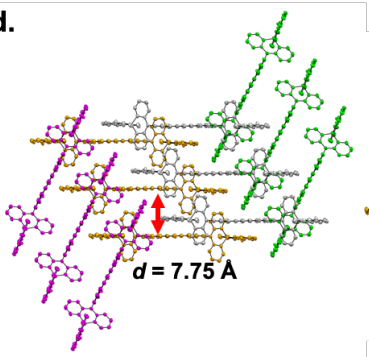

g.

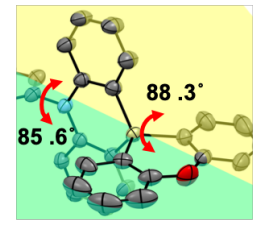

h.

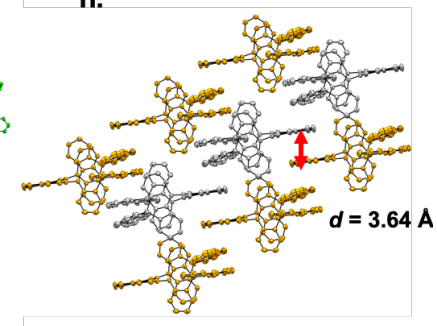

Fig. 2 The ORTEP drawings of $\mathbf{3}$ and $\mathbf{6}$ obtained by the Xray crystallographic analysis (thermal ellipsoids set at $50 \%$ probability; hydrogen atoms are omitted for clarity). a) Molecular structure, b) side view, c) an enlarged view of the donor, and d) packing structure of 3 ; e) Molecular structure, f) side view, g) an enlarged view of the donor, and $h$ ) packing structure of 6 .

\section{UV-vis absorption and steady-state PL spectra of diluted solutions}

To investigate the effect of Si substituents and regioisomerism on the photophysics of the D-A-D compounds, UV-vis absorption and steady-state photoluminescence $(\mathrm{PL})$ spectra of diluted solutions of 2-6 were measured. A representative spectra are shown in Fig. 3 (for the full detailed spectra, see the Fig. S1 in the ESI). The regioisomeric factor much more affects the photophysics in solution than the substituents on the $\mathrm{Si}$ center. Taking 3,11-isomer 1 and 2,12-isomer 4 as examples, the CT absorption band is slightly broader and weaker for $\mathbf{4}$ than $\mathbf{1}$ (Fig. 3a and b). The UV-vis absorption spectra within the same regioisomeric scaffold are almost the same, indicating little substituent of the $\mathrm{Si}$ center on the nature of electronic transitions (Fig. S1 in the ESI). Interestingly, solvatochromic behavior of regioisomers was quite different. In a non-polar solvent (cyclohexane), the 2,12-regioisomer 4 showed green emission ( $\lambda_{\mathrm{em}} 518 \mathrm{~nm}, \Phi_{\mathrm{PL}} 0.35$ ), which is red-shifted when compared with that of the 3,11-counterpart 1 ( $\lambda_{\mathrm{em}} 472 \mathrm{~nm}, \Phi_{\mathrm{PL}}$ 0.25) (Fig. 3a and b). Both compounds showed significant positive solvatoluminochromism as a function of permittivity of organic solvent, indicating CT nature in the excited state (Fig. 3). However, it is noted that in a highly polar solvent such as DMF, the PL spectrum of 4 showed in a bluer region ( $\lambda_{\mathrm{em}} 601 \mathrm{~nm}, \Phi_{\mathrm{PL}}$ 0.13 ) than that of 1 ( $\lambda_{\mathrm{em}} 685 \mathrm{~nm}, \Phi_{\mathrm{PL}}$ 0.02). Comparison of the Mataga-Lippert plots of 1-6 gave us an interesting insight into the regioisomeric effect on the steady-state photophysics (Fig. 3c). The slopes for the 3,11-regioisomers 1-3 are much steeper than those of 2,12-regioisomers 4-6, indicating the much more change in the dipole moment from the ground state $\left(\mu_{\mathrm{g}}\right)$ to the excited state $\left(\mu_{\mathrm{e}}\right)$ for the 3,11-regioisomers than the 2,12regioisomers. Within the same substitution pattern, there is little effect on the emission behavior including the PL spectra (Fig. S1 in the ESI). Another interesting tendency includes the larger intercepts in the plot for the 2,12-isomers than for the 3,12-isomers (Fig. 3c), which would be related to structural change in the excited states. This was partly supported by the theoretical calculation results that the 2,12-isomers drastically change the most stable conformation (eq-eq) from the ground state to the excited state (eq-ax, see the theoretical part).

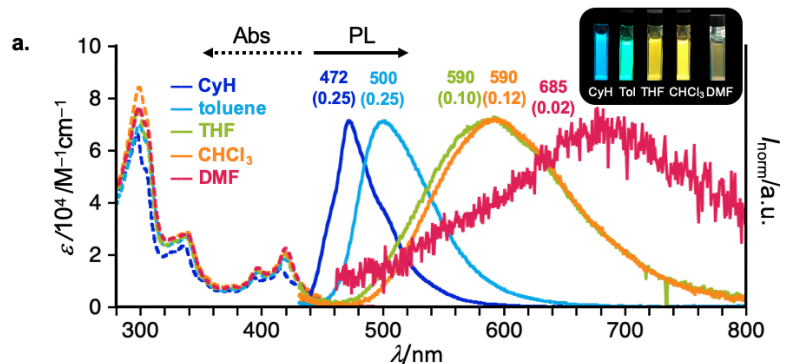

b.
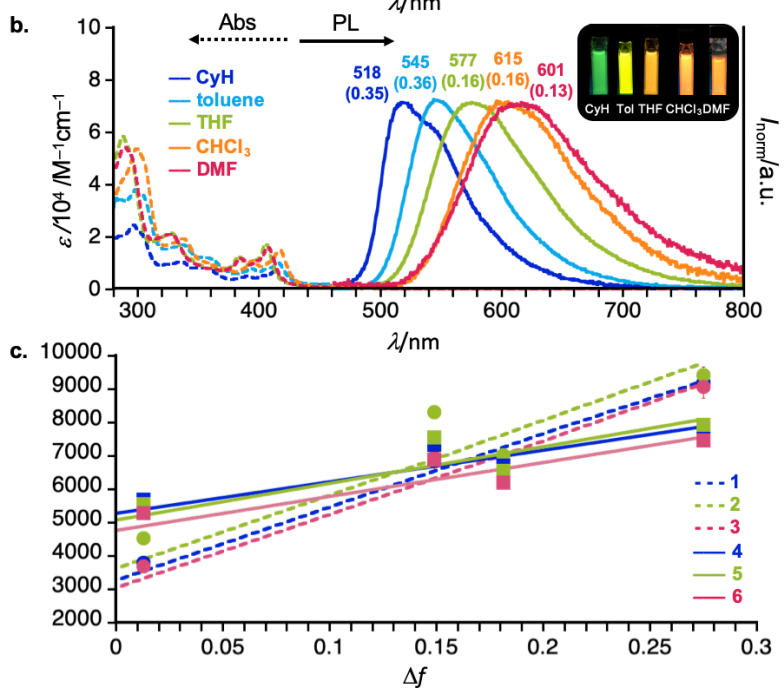

Fig. 3 UV-vis absorption and PL spectra of diluted solutions $\left(c=10^{-5} \mathrm{M}\right)$ of a) $\mathbf{1}$ and $\left.b\right) \mathbf{4}$. The values on the PL spectra and in parentheses indicate the emission maximum (nm) and photoluminescence quantum yield (PLQY) determined by integrating sphere. The excitation for PL measurements were conducted at $\lambda_{\mathrm{ex}} 420 \mathrm{~nm}$ for 1 and $\lambda_{\mathrm{ex}}$ $412 \mathrm{~nm}$ for 4 . The inset photos are taken under the irradiation of a UV lamp $\left(\lambda_{\mathrm{ex}} 420 \mathrm{~nm}\right)$. c) The MatagaLippert plots of D-A-D compounds 1-6. 


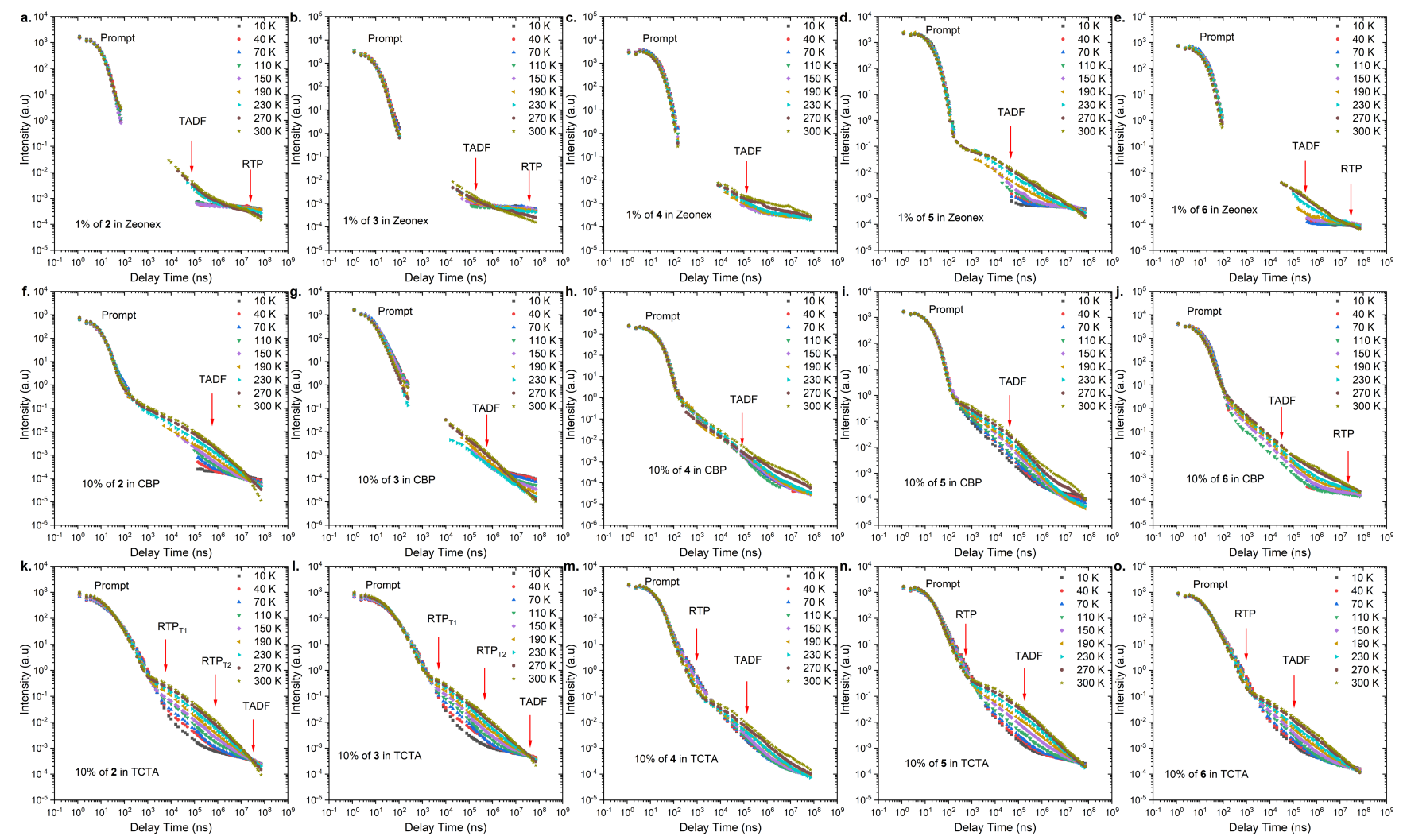

Fig. 4 Photophysical properties of compounds 2-6 in a different matrix. Intensity vs. delay time at different temperatures in various hosts. a) 2, b) 3, c) 4, d) 5, and e) 6 (1 wt\%) in Zeonex ${ }^{\circledR}$; f) 2, g) 3, h) 4, i) 5, and j) 6 (10 wt\%) in CBP; k) 2, l) 3, m) 4, n) 5, and o) 6 (10wt\%) in TCTA host.
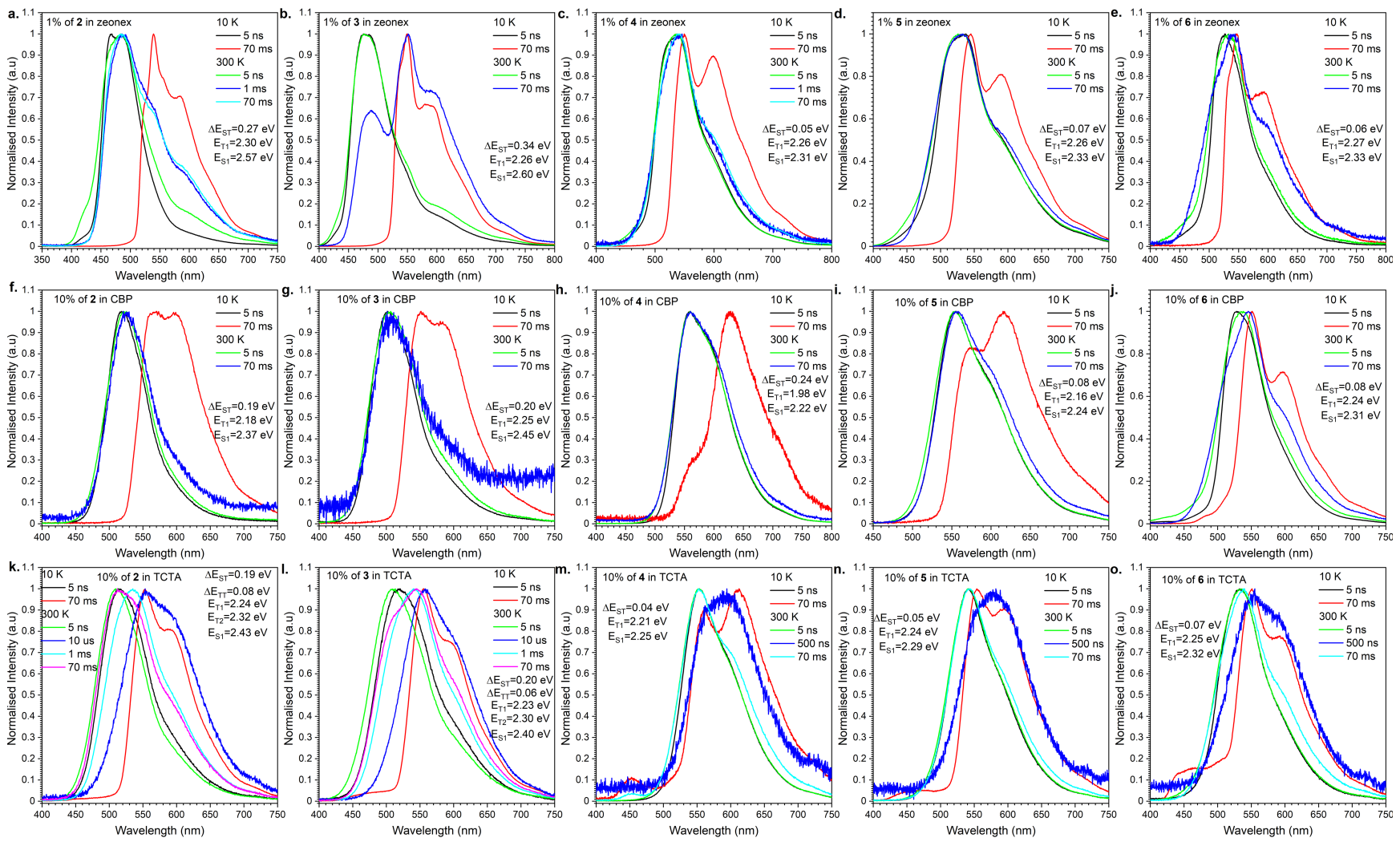

Fig. 5 Time-Resolved Spectra of compounds 2-6 in different matrix obtained during the intensity vs. delay time measurement

(Fig. 4). The energies correspond to the maximum emission peaks. a) 2, b) 3, c) 4, d) 5, and e) $\mathbf{6}\left(\mathbf{1}\right.$ wt\%) in Zeonex ${ }^{\circledR}$; f) $\mathbf{2}$, g) 3, h)

4, i) 5, and j) 6 (10 wt\%) in CBP; k) 2, l) 3, m) 4, n) 5, and o) 6 (10 wt\%) in TCTA host. 


\section{Time-resolved spectroscopic analysis}

To understand the actual recombination processes involved in the light generation, more detailed photophysical studies were conducted (Fig. 4 and Fig. 5). The photofunctional parameters are summarized in Table 1 . The analysis revealed the influence caused by not only molecular structure but also host material on the observed emission processes. Depending on the combination of the molecular structure and host material, TADF, RTP, dual TADF \& RTP, or dual RTP from the $T_{1}$ and $T_{2}$ states were observed (Fig. 4). As the analysis of emission change after the excitation with laser, the PL emission intensity versus a particular delay time in Zeonex ${ }^{\circledR}$ matrix showed emissions associated with dual TADF \& RTP processes for all the compounds (Fig. 4a-e). The transient intensity curves displayed a classical behavior of TADF and RTP process for compounds $\mathbf{2}$, 3, 5 and 6, where the delay component in $\mu$ s delay region increased as a function of temperature (TADF process), and the component in ms delay region decreased as a function of temperature (phosphorescence process). It should be noted that the long delayed component (phosphorescence) was not technically observed for compound 2,12-regioisomer 4, probably due to the overlap with long-lived TADF emission. Nevertheless, only the compound 3 based on 3,11-substituted D-A-D scaffold showed significant contributions of RTP emission ( $>50 \%$, Fig. $4 \mathrm{~b}$ and $5 \mathrm{~b}$ ). In the case of compounds 2 and 6, the RTP contribution is above $1 \%$ (Fig. $5 a$, e) and lower than $1 \%$ for compounds 4 and 5 (Fig. 5c, d).

For the purpose of application study, further time-resolved spectroscopic analysis in an OLED host was conducted (Fig. $4 \mathrm{f}-$ $\mathrm{o}$, and $5 \mathrm{f}-\mathrm{O})$. Based on our previous study, ${ }^{17}$ we compared the behavior of the emitters in CBP $\left[4,4^{\prime}\right.$-bis $\left(N\right.$-carbazolyl)-1, $1^{\prime}$ biphenyl] and TCTA [4,4',4"-tri(9-carbazoyl)triphenylamine] matrix. In the case of behavior in CBP host, all the emitters except for 6 exhibited TADF emission at $300 \mathrm{~K}$ (Fig. 4f-i and Fig. $5 f-i)$. The emission intensity versus delay time curves of compounds 2-5 changed with temperature in the way that delay component related with TADF process increased as a function of temperature whereas the phosphorescence in $\mathrm{ms}$ delay regime decreased and disappeared above $150 \mathrm{~K}$ (Figure $4 \mathrm{f}-\mathrm{i})$. The emission spectra at $300 \mathrm{~K}$ for compounds $\mathbf{2}-\mathbf{5}$ at the delay time of $70 \mathrm{~ms}$ are nicely overlapped with the prompt emission and significantly different from the phosphorescence spectra, indicating the emission of $\mathbf{2}-\mathbf{5}$ in CBP is radiated from the $S_{1}$ state. In all the cases, the emission spectra are not wellresolved (i.e., Gaussian shape), suggesting the $S_{1}$ state is ascribed to ${ }^{1} \mathrm{CT}$ state (Figure $5 \mathrm{f}-\mathrm{i}$ ). It is noted that a different type of emission was observed for 2,12-regioisomeic spiro compound $\mathbf{6}$. At a very long delay time, mixed emission of TADF and RTP processes were observed (Figure $5 \mathrm{j}$ ).

Most importantly, unusual behavior was observed in TCTA matrix (Fig. 4k-o and Fig. 5k-o). The 3,11-regioisomers 2 and 3 exhibited dual RTP emissions radiated from the $T_{1}$ and $T_{2}$ states, which are quite short-lived (in $\mu$ s order) as for phosphorescence (Fig. 4k, l, and Table 1). Also, TADF occurred, but at very long delay times (Fig. 5k,I, and Fig. S2 in the ESI). In contrast, the 2,12regioisomers compounds 4-6 displayed dual TADF \& RTP emissions, but again surprisingly the RTP is much shorter-lived than TADF (Fig. 4m, n, o, and Table 1). It seems that the RTP occurs below $1 \mu \mathrm{s}$, and thus, we observed the decrease of phosphorescence with the increase of temperature (Fig. $4 \mathrm{~m}, \mathrm{n}$, and o). The shape of the delayed emission spectra corresponds to those acquired at a low temperature at $70 \mathrm{~ms}$ delayed phosphorescence (Fig. S3 in the ESI). The TADF emissions for compounds 4-6 (2,12-regioisomer) started to be visible after 10 $\mu$ delay time and are long-lived, probably due to the extensive ISC/rISC cycling (Fig. 5m, n, o).

\section{Thermal stability}

To fabricate OLEDs by thermal evaporation technique, the thermal stability of the synthesized compounds was investigated with thermogravimetry analysis (TGA, for the detailed data on the TGA profiles, see the Fig. S4 and S5 in the

Table 1. Summary of the general photophysical properties of the compounds.

\begin{tabular}{|c|c|c|c|c|c|c|c|c|c|c|c|c|c|c|}
\hline Comp. & $\begin{array}{l}\lambda_{\mathrm{em}}{ }^{a} \\
(\mathrm{~nm})\end{array}$ & Host & $\Phi_{02} / \Phi_{\mathrm{deg}}^{\mathrm{b}}$ & $\begin{array}{l}\tau_{\mathrm{PF}}{ }^{\mathrm{c}} \\
(\mathrm{ns})\end{array}$ & $\begin{array}{l}\tau_{\mathrm{DF}}^{d} \\
(\mu \mathrm{s})\end{array}$ & $\begin{array}{l}\tau_{\mathrm{PH} 1} e \\
(\mu \mathrm{s})\end{array}$ & $\begin{array}{l}\tau_{\mathrm{PH} 2}{ }^{e} \\
(\mathrm{~ms})\end{array}$ & $D F / P F^{f}$ & $\begin{array}{c}E_{\mathrm{a}^{g}} \\
(\mathrm{eV})\end{array}$ & $\begin{array}{l}\mathrm{S}_{1}{ }^{h} \\
(\mathrm{eV})\end{array}$ & $\begin{array}{l}\mathrm{T}_{1}^{h} \\
(\mathrm{eV})\end{array}$ & $\begin{array}{l}\mathrm{T}_{2}{ }^{h} \\
(\mathrm{eV})\end{array}$ & $\begin{array}{l}\Delta E_{\mathrm{ST}}{ }^{i} \\
(\mathrm{eV})\end{array}$ & $\begin{array}{l}\Delta E_{\pi^{i}} \\
(\mathrm{eV})\end{array}$ \\
\hline \multirow{3}{*}{2} & 483 & Zeonex & $0.065 / 0.098$ & $6.9 \pm 0.3$ & $7.8 \pm 0.3$ & - & - & 2.31 & 0.03 & 2.57 & 2.30 & - & 0.27 & - \\
\hline & 523 & CBP & $0.186 / 0.372$ & $6.8 \pm 0.2$ & $1.5 \pm 0.1$ & - & - & 3.80 & 0.06 & 2.37 & 2.18 & - & 0.19 & - \\
\hline & 527 & TCTA & $0.122 / 0.337$ & $16.3 \pm 0.7$ & $\begin{array}{c}8777.9 \pm \\
689.3\end{array}$ & $3.8 \pm 0.4$ & $\begin{array}{c}0.86 \pm \\
0.03\end{array}$ & 3.76 & 0.04 & 2.43 & 2.24 & 2.32 & 0.19 & 0.08 \\
\hline \multirow{3}{*}{3} & 477 & Zeonex & $0.065 / 0.072$ & $6.5 \pm 0.2$ & $26.4 \pm 1.7$ & $\begin{array}{c}227107.8 \pm \\
2322.1\end{array}$ & - & 1.11 & 0.02 & 2.60 & 2.26 & - & 0.34 & - \\
\hline & 505 & CBP & $0.153 / 0.168$ & $5.8 \pm 0.2$ & $6.0 \pm 0.6$ & - & - & 0.87 & 0.03 & 2.45 & 2.25 & - & 0.21 & - \\
\hline & 536 & TCTA & $0.094 / 0.223$ & $27.8 \pm 1.2$ & $\begin{array}{c}13724.7 \pm \\
1182.2\end{array}$ & $2.3 \pm 0.2$ & $\begin{array}{c}1.17 \pm \\
0.07\end{array}$ & 3.11 & 0.03 & 2.44 & 2.23 & 2.30 & 0.20 & 0.06 \\
\hline \multirow{3}{*}{4} & 536 & Zeonex & $0.274 / 0.307$ & $14.4 \pm 0.5$ & $29.1 \pm 1.0$ & - & - & 0.75 & 0.03 & 2.31 & 2.26 & - & 0.05 & - \\
\hline & 559 & CBP & $0.387 / 0.439$ & $13.7 \pm 0.6$ & $2.0 \pm 0.2$ & - & - & 0.54 & 0.04 & 2.22 & 1.98 & - & 0.24 & - \\
\hline & 553 & TCTA & $0.518 / 0.550$ & $15.6 \pm 0.7$ & $5.4 \pm 0.6$ & $0.54 \pm 0.05$ & - & 0.93 & 0.03 & 2.25 & 2.21 & - & 0.03 & - \\
\hline \multirow{3}{*}{5} & 525 & Zeonex & $0.063 / 0.118$ & $13.2 \pm 0.6$ & $4.0 \pm 0.4$ & - & - & 1.21 & 0.03 & 2.36 & 2.27 & - & 0.08 & - \\
\hline & 554 & CBP & $0.325 / 0.354$ & $12.3 \pm 0.6$ & $8.6 \pm 0.9$ & - & - & 1.70 & 0.02 & 2.24 & 2.16 & - & 0.08 & - \\
\hline & 541 & TCTA & $0.222 / 0.295$ & $14.5 \pm 0.7$ & $18.5 \pm 0.9$ & $0.48 \pm 0.05$ & - & 3.18 & 0.04 & 2.29 & 2.24 & - & 0.05 & - \\
\hline \multirow{3}{*}{0} & 533 & Zeonex & $0.093 / 0.103$ & $10.2 \pm 0.6$ & $33.5 \pm 5.9$ & $12138.6 \pm 391.2$ & - & 1.27 & 0.04 & 2.33 & 2.27 & - & 0.06 & - \\
\hline & 535 & CBP & $0.258 / 0.278$ & $8.3 \pm 0.2$ & $2.9 \pm 0.3$ & $1914.3 \pm 59.7$ & - & 1.55 & 0.03 & 2.31 & 2.24 & - & 0.08 & - \\
\hline & 533 & TCTA & $0.171 / 0.203$ & $14.7 \pm 0.7$ & $4.3 \pm 0.3$ & $0.26 \pm 0.02$ & - & 2.70 & 0.04 & 2.32 & 2.25 & - & 0.07 & - \\
\hline
\end{tabular}

${ }^{a}$ Photoluminescence maximum; ${ }^{b}$ Photoluminescence quantum yield in air-equilibrated and degassed; ${ }^{c}$ Prompt fluorescence lifetime; ${ }^{d}$ Delayed fluorescence lifetime; ${ }^{e}$ Room-temperature phosphorescence lifetime from $\mathrm{T}_{1}$ or $\mathrm{T}_{2}$ triplet energy level; ${ }^{f}$ Delayed fluorescence (DF) to prompt fluorescence $(\mathrm{PF})$ ratio in $\mathrm{MCH}$ as indicated from decay profile; ${ }^{g}$ Activation energy of the triplet to singlet transfer. Error \pm 0.01 eV.; ${ }^{h}$ Singlet and triplet energy in. Error $\pm 0.03 \mathrm{eV} ;{ }^{i}$ Energy splitting. Error $\pm 0.05 \mathrm{eV}$. 
ESI). When compared with the degradation temperature $T_{d}(5$ wt\%) of compound 1 [ $T_{\mathrm{d}}\left(5 \mathrm{wt} \%\right.$ under $\left.\mathrm{N}_{2}\right)=496{ }^{\circ} \mathrm{C}$ ], the $T_{\mathrm{d}}(5$ wt\% under $\mathrm{N}_{2}$ ) of the 2,12-regioisomer 4 was found slightly low $\left(451^{\circ} \mathrm{C}\right)$. There is a tendency that $T_{\mathrm{d}}$ of 2,12-isomer is lower than that of the corresponding 3,11-isomer (see the ESI), possibly due to the involvement of thermal reaction between spatiallyclose donor units. Substituent effect on the thermal stability was clearly observed: in the 3,11-regioisomeric scaffold, the steric stiffness allows for increasing $T_{\mathrm{d}}\left(5 \mathrm{wt} \%\right.$ under $\mathrm{N}_{2}$ ) [3 (521 $\left.\left.{ }^{\circ} \mathrm{C}\right)>\mathbf{1}\left(496{ }^{\circ} \mathrm{C}\right)>2\left(440{ }^{\circ} \mathrm{C}\right)\right]$. This is the case with the 2,12regioisomers $\left[6\left(483^{\circ} \mathrm{C}\right)>\mathbf{4}\left(451^{\circ} \mathrm{C}\right)>\mathbf{5}\left(332^{\circ} \mathrm{C}\right)\right]$.

To investigate the electrochemical stability of the emitters, cyclic voltammetry (CV) of dichloromethane (DCM) solutions of compounds 2-5 were performed (Fig. S6 in the ESI). All the compounds displayed a (quasi)reversible reduction process at around ${ }^{r e d} E_{\text {onset }}-1.79-1.50 \mathrm{eV}\left(\mathrm{vs} \mathrm{Fc} / \mathrm{Fc}^{+}\right.$) and an irreversible oxidation at around ${ }^{\circ} E_{\text {onset }}+0.55-0.89 \mathrm{eV}\left(\mathrm{vs} F \mathrm{Fc} / \mathrm{Fc}^{+}\right)$. The estimated ionization potential (IP) and electron affinity (EA) of the compounds were estimated. The IPs follow the order of $\mathbf{6}(5.99 \mathrm{eV})>\mathbf{3}(5.89 \mathrm{eV})$ $>\mathbf{1}(5.85 \mathrm{eV})^{17} \sim \mathbf{2}(5.84 \mathrm{eV})>\mathbf{4}(5.65 \mathrm{eV}) \sim \mathbf{5}(5.63 \mathrm{eV})$, while the EAs follow the order of $\mathbf{6}(3.60 \mathrm{eV})>\mathbf{2}(3.52 \mathrm{eV}) \sim 3(3.50 \mathrm{eV}) \sim 1$ (3.49 $\mathrm{eV})^{17}>\mathbf{5}(3.36 \mathrm{eV})>\mathbf{4}(3.31 \mathrm{eV})$.

\section{Fabrication and evaluation of OLEDs devices}

As the final experimental study, the OLED devices were fabricated and investigated the performance (Fig. 6). The behavior of the emitters in two hosts, CBP and TCTA, was characterized in OLED devices. The structures of the fabricated OLEDs are illustrated in Fig. 6: Devices 1-5 -ITO/NPB [N, $N^{\prime}-\mathrm{di}(1-$ naphthyl)- $N, N^{\prime}$-diphenyl-(1,1'-biphenyl)-4,4'-diamine] $n \mathrm{n}) /$ TSBPA $\left[4,4^{\prime}\right.$-(diphenylsilanediyl)bis( $N, N$-diphenylaniline) $]$ $(10 \mathrm{~nm}) / 10 \% 2,3,4,5$ or 6 in CBP $(30 \mathrm{~nm}) / T P B i\left[2,2^{\prime}, 2^{\prime \prime}-(1,3,5-\right.$ benzinetriyl)-tris(1-phenyl-1-H-benzimidazole)] $(60 \mathrm{~nm}) / \mathrm{LiF}(1$ $\mathrm{nm}) / \mathrm{Al}(100 \mathrm{~nm})$; Devices 6-10- ITO/NPB (40 nm)/TAPC [4,4'cyclohexylidenebis( $N, N$-bis(4-methylphenyl)benzenamine)] (10 $\mathrm{nm}) / 10 \%$ 2, 3, 4, 5 or 6 in TCTA $(30 \mathrm{~nm}) / \mathrm{TPBi}\left[2,2^{\prime}, 2^{\prime \prime}-(1,3,5-\right.$ benzinetriyl)-tris(1-phenyl-1-H-benzimidazole)] $(60 \mathrm{~nm}) / \mathrm{LiF}(1$ $\mathrm{nm}) / \mathrm{Al}(100 \mathrm{~nm})$ (Fig. 6).
All the devices fabricated with the emitters in CBP host showed TADF. It is noted that even 2,12-regioisomer 6, which showed dual TADF \& RTP emission in the photophysical analysis in blended films (Fig. 5j), displayed only TADF in OLED (Fig. 6a).

More interesting phenomenon was observed in the electroluminescence spectra of the OLED devices fabricated with TCTA host. Devices 6 and 7 with compounds 2 and 3 (3,11regioisomers) showed efficient RTP emission from the $T_{2}$ and $T_{1}$ excited state, probably due to the fact those excited states were much shorter-lived than TADF (Fig. 4 k, I, Fig. 5k, I, and Fig. S7b,c). As for compound 4 (2,12-regioisomer), device 8 displayed again dual RTP \& TADF electroluminescence (Fig. 6e and Fig. S7d). Finally, the devices 9 and 10 based on the emitters 5 and $\mathbf{6}(2,12$ regioisomers) had electroluminescence emission through RTP process (Fig. 6e and Fig. S7e, f). The TADF process was unfavourable process, probably because of longer lifetime than RTP process (Table 1).

The characteristics of the OLED devices revealed a significant increase of OLED efficiency depending on the host used (Fig. 6c, d, g, h). The device based on CBP was found be the most efficient in all of the cases except for compound $3(3,11-$ regioisomer), where efficiency in TCTA was $50 \%$ higher (Fig. 6c, g). The highest external quantum efficiency (EQE) was achieved with the OLED fabricated with compound 4 (2,12-regioisomer) in both of the hosts (12.6\% in CBP; $11.2 \%$ in TCTA). But, in both cases, the majority of emission comes from TADF process (Fig. S7d). Most importantly, the highest RTP-related OLED was obtained for devices 6 and 9 based on compounds 2 and 5 in TCTA (7.4\% and $6.85 \%)$, which are higher than that of $1 .{ }^{17}$ This suggested that the EQEs are less likely dependent on the regioisomeric influence but mostly on the donor effect. The highest luminance was obtained for the OLED based on compound 2 in CBP, up to $\left(54,800 \mathrm{~cd} / \mathrm{m}^{2}\right)$, where in TCTA host it was for device based on compound $4\left(51,100 \mathrm{~cd} / \mathrm{m}^{2}\right.$, Fig. $6 \mathrm{~d}$, h).

\section{Theoretical calculations}

To better understand the mechanism behind the different behavior observed for 3,11 and 2,12-isomers, we resorted to
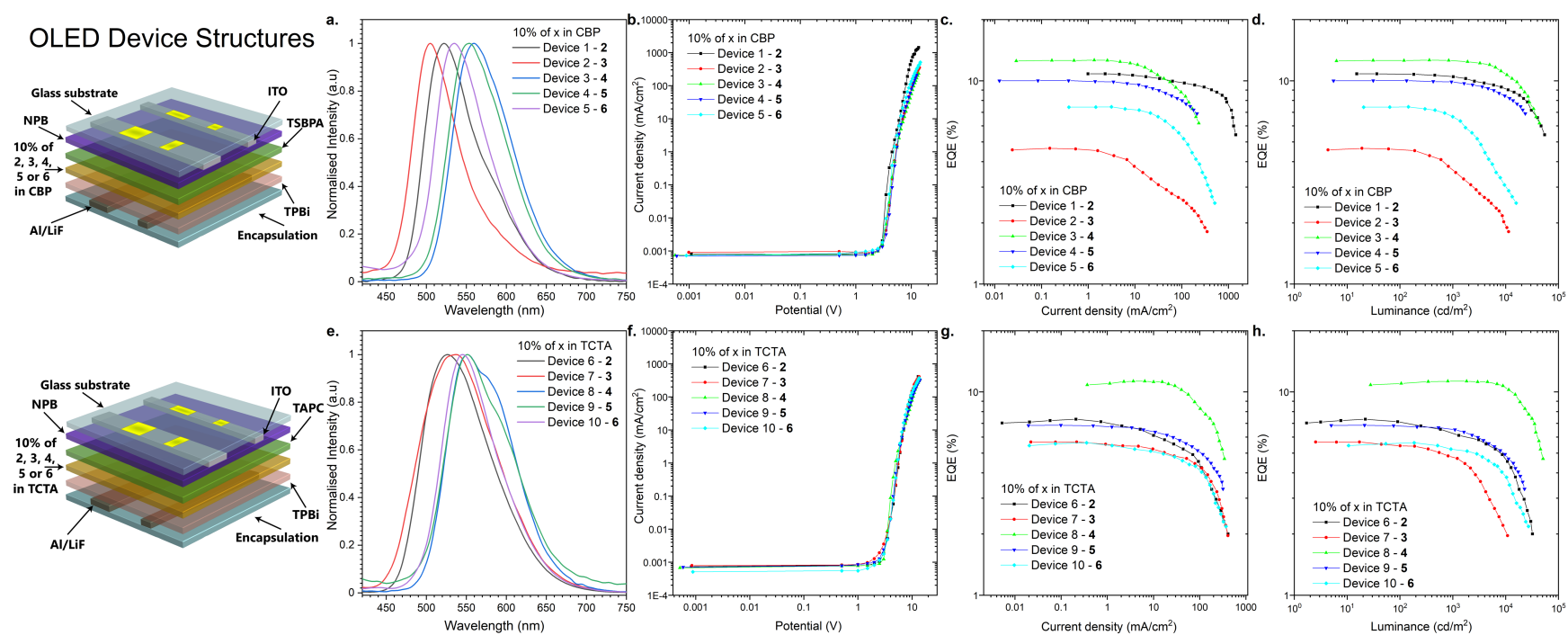

Fig. 6 The characteristics of the OLED devices. a), e) Electroluminescence spectra. b), f) Current density-bias characteristics. c), g) EQE-current density characteristics. d), h) EQE - luminance characteristics. 
a.

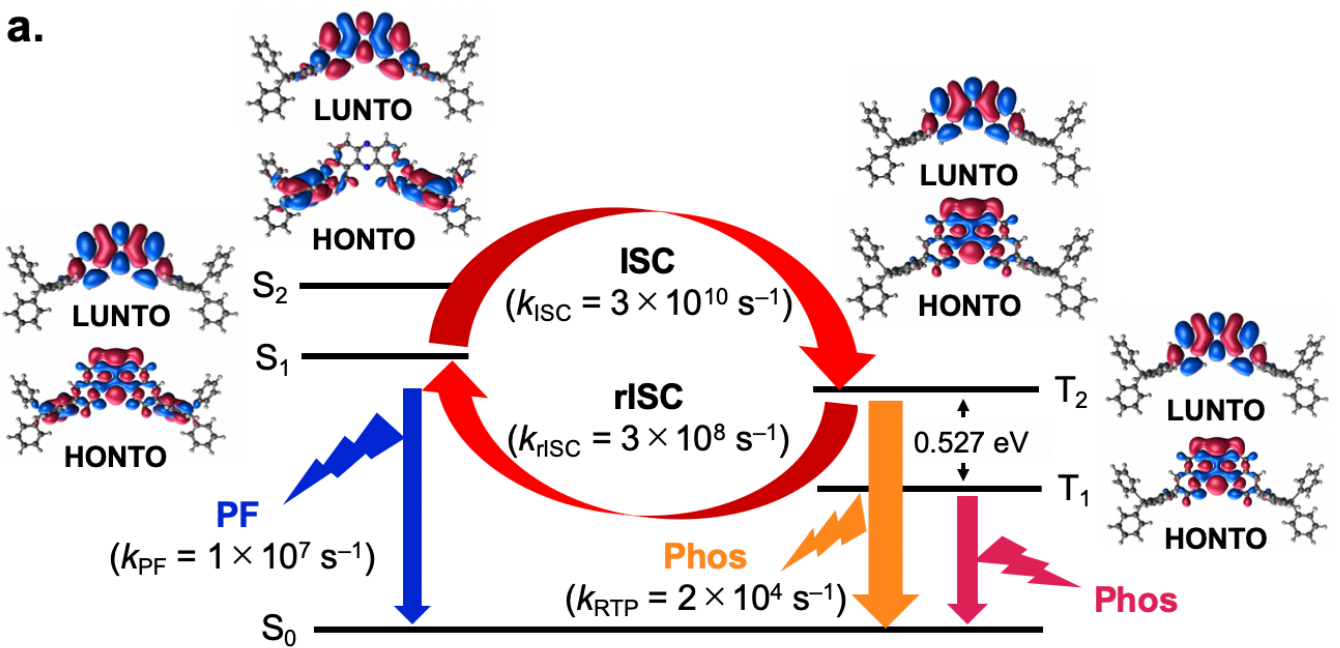

b.

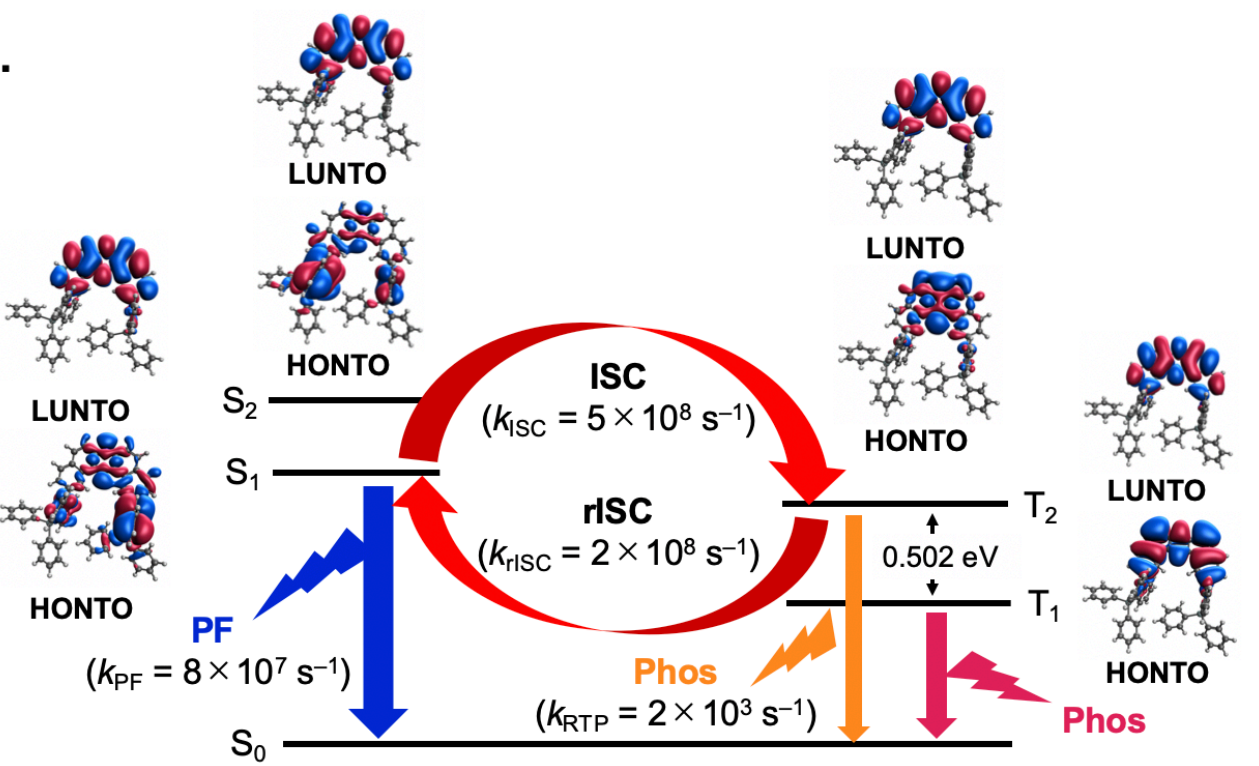

Fig. 7 Schematics showing the NTOs for the first two singlet and triplet states as well as rate estimates for different photophysical processes for compounds a) $\mathbf{1}$ and b) $\mathbf{4}$ calculated with the ground state geometry. ISC rates correspond to the sum of rates from $S_{1}$ to the first 5 triplet states.

electronic structure calculations, details of which can be found in ESI. DFT calculations were performed to determine equilibrium geometries and normal mode frequencies for each of the molecules in the eq-eq, eq-ax and ax-ax conformations. These calculations reveal that in the ground state, all molecules display a preference for the eq-eq conformation, in particular compounds $\mathbf{2}$ and $\mathbf{3}$ (see Table S4-S9 in the ESI). However, when considering the optimized geometries in the $S_{1}$ state, conformational preference is shifted towards eq-ax conformations in the case of 2,12-isomers, but remains eq-eq for 3,11-isomers. This constitutes a key difference between both kinds of molecules, and it is responsible for their different photophysical behavior. In Fig. 7, natural transition orbitals (NTOs) are shown for the $S_{1}, S_{2}, T_{1}$ and $T_{2}$ states of two molecules representative of 2,12- and 3,11-regioisomers, namely compounds $\mathbf{1}$ (Fig. 7a) and $\mathbf{4}$ (Fig. 7b) in the ground state geometry. It can be seen that for the 3,11-isomer (1) the $S_{1}$ state corresponds to a mixture of charge transfer (CT) and local excitation (LE), whereas the $S_{2}$ has a clear CT character (Fig. 7a). Solvent induced stabilization of the $S_{2}$ state is expected to reduce its energy difference with respect to $S_{1}$, in particular in more polar solvents. In contrast with compound $\mathbf{1}$, the 2,12isomer 4 shows $S_{1}$ and $S_{2}$ states with a mixed character between CT and LE (Fig. 7b). As for the triplet states, both $T_{1}$ and $T_{2}$ display mostly a LE character regardless of the isomer in question (Fig. 7a, b).

To investigate conformational influence on the photophysical properties, fluorescence and phosphorescence spectra were simulated and ISC rates were estimated using the nuclear ensemble method as implemented in the NEMO software (Table S8-S10 in the ESI). ${ }^{20}$ As it can be seen in Table S10 in the ESI, fluorescence from 2,12-isomers would be blue shifted if emitted from eq-eq conformations. These results show that the difference in conformational preference accounts for the red shift of the fluorescence in 2,12-isomers when compared to their 3,11-counterparts. In addition, fluorescence 
rates from the eq-ax conformers of the 2,12-isomers are consistently higher than those of the eq-eq conformers of 3,11 isomers, sometimes by as much as one order of magnitude, as shown in Table S10 and S11 in the ESI and in Fig. 7. This fact explains the higher PLQY observed for compound $\mathbf{4}$ in comparison to compound $\mathbf{1}$, as observed in Fig. 3.

When it comes to the role of triplet states, the estimation of ISC rate show that for all molecules, regardless of conformation, ISC is not efficient between $S_{1}$ and $T_{1}$, primarily due to high energy gaps between these states. Analysis of the rate estimates indicate rather that ISC is expected to take place mostly between $S_{1}$ and $T_{2}$ states (Table S13-S15 in the ESI). Higher energy triplet states can also take part in ISC, but low energy gaps with respect to lower lying triplets make internal conversion back to $T_{2}$ a likely result. On the other hand, average gaps between $T_{2}$ and $T_{1}$ states are high ( $>0.4 \mathrm{eV}$, as seen in Table S16 in the ESI), which should hinder reverse internal conversion (rIC) and allow continuous interconversion between triplet and singlet excitons by means of the $S_{1}-T_{2}$ pathway. This is true for all molecules analyzed here, which prompts the question of why the 3,11-isomers display more efficient RTP whereas the 2,12isomers display mostly TADF. Any emission process, whether it be fluorescence from $S_{1}$ or phosphorescence from $T_{2}$, has to compete against the different available ISC processes, some of which are highly efficient. As mentioned above, fluorescence rates from the eq-ax conformation are typically larger than those from their eq-eq counterparts. Since the 2,12-compounds have a conformational preference for eq-ax in the excited state, fluorescence can outcompete ISC, constituting thus a possible pathway for exciton relaxation and explaining the observation of TADF in these molecules. On the other hand, 3,11-isomers, with their lower fluorescence rates, have a higher chance of undergoing radiative emission when in the $T_{2}$ state, where this process competes against the less efficient up-conversion from $T_{2}$ to $S_{1}$. Furthermore, rISC rates from $T_{2}$ to $S_{1}$ in the 2,12-isomers are typically higher than in their 3,11-counterparts, which makes TADF more efficient as well. For these reasons, more significant RTP is observed from the 3,11-isomers. It is also worth mentioning that the estimated phosphorescence rates from the $T_{2}$ states of the molecules in this work are 1-3 orders of magnitude larger than phosphorescence rates from their $T_{1}$ states, which further emphasizes the role of emission from $T_{2}$ in the photophysics of these compounds.

\section{Conclusions}

In summary, we have developed new family of DBPHZ-cored DA-D type organic emitters that show TADF, RTP, and both, depending on the conditions. The developed emitters were applied to OLED devices, which successfully achieved a high EQE up to $7.4 \%$ for RTP-based OLED. Using the series of the developed emitters, the systematic structure-property relationship study was conducted to reveal the effect of regioisomerism on the fate of the excited states of the organic molecules. Although the detailed photophysical processes seem complex, a main fate of the excited state of the developed DBPHZ-cored D-A-D compounds is significantly governed by the donor positions. Theoretical calculations revealed a significant difference in conformational preference between the ground and the excited states, which would also affect the rate of photophysical process involved. Especially, the implication of the main role of $\mathrm{T}_{2}$ state in the ISC/rISC recycling process would allow for expanding the horizon of organic emissive materials that utilize excited triplet states in the future.

\section{Conflicts of interest}

There are no conflicts to declare.

\section{Acknowledgements}

Y.T. acknowledges a Grant-in-Aid for Scientific Research on Innovative Area "Aquatic Functional Materials: Creation of New Materials Science for Environment-Friendly and Active Functions" (JSPS KAKENHI Grant Number JP19H05716) from the MEXT (Ministry of Education, Culture, Science and Technology, Japan), a Grant-in-Aid for Scientific Research (B) (JSPS KAKENHI Grant Number JP2OH02813), a Grant-in-Aid for Challenging Research (Exploratory) (JSPS KAKENHI Grant Number JP21K18960), and the Continuation Grants for Young Researchers from the Asahi Glass Foundation, and the Research Grant in the Natural Science from the Mitsubishi Foundation. Y.T. and S.M. acknowledge NIPPOH CHEMICALS for supplying $\mathrm{N}, \mathrm{N}$-diiodo-5,5-dimethylhydantoin (DIH). P. de S. and L. E. de S. acknowledge support by a research grant (00028053) from VILLUM FONDEN. P.D. and P.Z.C acknowledges the Polish National Science Centre funding, grant no. 2017/25/B/ST5/02488. P.D. and N.O.D. acknowledges the supporting awards from the Rector of the Silesian University of Technology (04/040/BKM20/0124, 04/040/RGJ21/0149). Y.T., N.O.D., P.Z.C., P. de S. and P.D. acknowledge the EU's Horizon 2020 for funding the OCTA project under grant agreement No 778158. Research work supported from the funds for science in 2018 - 2022 allocated to the implementation of an international co-financed project by the Polish Ministry of Education and Science. P.D. N.O.D. and P.Z.C. acknowledges the supporting actions from EU's Horizon 2020 ERA-Chair project ExCEED, grant agreement No 952008. Y.T. acknowledge Professor Hiroshi Miyasaka at Osaka University for fruitful discussion.

\section{References}

1 H. Uoyama, K. Goushi, K. Shizu, H. Nomura and C. Adachi, Nature, 2012, 492, 234-238.

2 H. Nakanotani, Y. Tsuchiya and C. Adachi, Chem. Lett., 2021, 50, 938-948.

3 Y. Liu, C. Li, Z. Ren, S. Yan and M. R. Bryce, Nat. Rev. Mater., 2018, 3, 1-20.

4 G. Bergamini, A. Fermi, C. Botta, U. Giovanella, S. Di Motta, F. Negri, R. Peresutti, M. Gingras and P. Ceroni, J. Mater. Chem. C, 2013, 1, 2717-2724.

5 G. Zhan, Z. Liu, Z. Bian and C. C. Huang, Front. Chem., 2019, 7, 305.

6 X. Yu, H. Zhang and J. Yu, Aggregate, 2021, 2, 20-34.

7 V.-N. Nguyen, A. Kumar, M. H. Lee and J. Yoon, Coord. Chem. Rev., 2020, 425, 213545. 
8 P. Data and Y. Takeda, Chem. Asian J., 2019, 14, 1613-1636.

9 F. B. Dias, T. J. Penfold and A. P. Monkman, Methods Appl Fluoresc, 2017, 5, 012001.

10 X.-K. Chen, D. Kim and J.-L. Brédas, Acc. Chem. Res., 2018, 51, $2215-2224$.

11 H. Ma, A. Lv, L. Fu, S. Wang, Z. An, H. Shi and W. Huang, Ann. Phys., 2019, 531, 1800482.

12 D. Sasikumar, A. T. John, J. Sunny and M. Hariharan, Chem. Soc. Rev., 2020, 49, 6122-6140.

13 G. Zhang, J. Chen, S. J. Payne, S. E. Kooi, J. N. Demas and C. L. Fraser, J. Am. Chem. Soc., 2007, 129, 8942-8943.

14 Y. Takeda, T. Kaihara, M. Okazaki, H. Higginbotham, P. Data, N. Tohnai and S. Minakata, Chem. Commun., 2018, 54, 68476850.

15 R. Huang, N. A. Kukhta, J. S. Ward, A. Danos, A. S. Batsanov, M. R. Bryce and F. B. Dias, J. Mater. Chem., 2019, 7, 13224-13234.

16 N. A. Kukhta and M. R. Bryce, Mater. Horiz., 2021, 8, 33-55.

17 H. F. Higginbotham, M. Okazaki, P. de Silva, S. Minakata, Y. Takeda and P. Data, ACS Appl. Mater. Interfaces, 2021, 13, 2899-2907.

18 Z. He, W. Zhao, J. W. Y. Y. Lam, Q. Peng, H. Ma, G. Liang, Z. Shuai and B. Z. Tang, Nat. Commun., 2017, 8, 416.

19 Y. Takeda, M. Okazaki and S. Minakata, Chem. Commun., 2014, 50, 10291-10294.

20 L. E. de Sousa and P. de Silva, J. Chem. Theory Comput., 2021, 17, 5816-5824; https://github.com/LeonardoESousa/NEMO 